\title{
Knockdown of Dock7 in vivo specifically affects myelination by Schwann cells and increases myelin thickness in sciatic nerves without affecting axon thickness
}

\author{
Tomohiro Torii $^{*}$, Yuki Miyamoto ${ }^{1 *}$, Motoshi Nagao ${ }^{1}$, Naoko Onami ${ }^{2}$, Hideki Tsumura ${ }^{2}$, \\ Masahiro Maeda ${ }^{3}$, Kazuaki Nakamura ${ }^{1}$, Akito Tanoue ${ }^{1}$, Junji Yamauchi, ${ }^{1,4 \#}$ \\ ${ }_{1}^{1}$ Department of Pharmacology, National Research Institute for Child Health and Development, Tokyo, Japan \\ ${ }^{2}$ Laboratory Animal Resource Facility, National Research Institute for Child Health and Development, Tokyo, Japan \\ ${ }^{3}$ Immuno-Biological Laboratories, Co., Ltd., Gunma, Japan \\ ${ }^{4}$ The Japan Human Health Sciences Foundation, Tokyo, Japan \\ Email: jyamauchi@nch.go.jp
}

Received 27 April 2012; revised 9 May 2012; accepted 29 May 2012

\begin{abstract}
During development of the peripheral nervous system (PNS), Schwann cells (SCs) wrap individual axons to form myelin sheaths, which act as surrounding insulators and markedly enhance the propagation of the action potential. In peripheral neuropathies such as Guillain-Barré syndrome (GBS) and inherited demyelinating Charcot-Marie-Tooth (CMT) disease and diabetic neuropathies, chronic demyelination and defective remyelination are repeated, causing more severe neuropathies. It is thus thought that development of a drug that promotes proper myelination with minimal side effects could provide an effective therapy for these diseases. As yet, however, little is known about therapeutic target molecules and genetically-modified mice for testing such approaches. We previously cloned the $d o c k 7$ gene and characterized Dock7 as the regulator controlling SC myelination; however, an important issue, whether knockdown of Dock7 specifically affects myelination by SCs but not leaves neurons unaffected, has remained unclear. Here, we generate newly-produced transgenic mice harboring short-hairpin RNA (shRNA) targeting Dock7. We also describe that Dock7 shRNA transgenic mice exhibit enhanced myelin thickness without affecting axon thickness in sciatic nerves of the PNS, as reduced thickness of the axon diameter is the primary indicator of denatured neurons. Similarly, purified in vitro SC-neuronal cocultures established from transgenic mice exhibit an enhanced formation of myelin segments, suggesting that knockdown of Dock7 promotes myelination by SCs. Collectively, Dock7 knockdown specifically affects SC myelination in
\end{abstract}

${ }^{*}$ These authors contributed equally to this work.

\#Corresponding author. sciatic nerves, providing evidence that Dock7 may be a promising drug-target-specific molecules for developing a therapy for peripheral neuropathies that aims to enhance myelination.

Keywords: Dock7; Transgenic Mouse; Schwann Cell; Myelination; Axon Diameter

\section{INTRODUCTION}

The myelin sheath is a unique multiple-layer structure that acts as an insulator between surrounding axons, and markedly enhances the propagation of the action potential $[1,2]$. The myelin sheath is derived from myelinforming glial cells, Schwann cells (SCs) in the peripheral nervous system (PNS) and oligodendrocytes (OLs) in the central nervous system (CNS). Over time, myelin sheaths grow to more than 100 times larger than the collective surface area of premyelinating SC or OL plasma membranes $[1,2]$. On the other hand, inherited neuropathies such as Charcot-Marie-Tooth (CMT) disease, GuillainBarré syndrome (GBS), and certain diabetic neuropathies cause chronic demyelination, resulting in repeated cycles of demyelination and defective remyelination that cause more severe neuropathies [3-5]. These major demyelinating diseases are primarily peripheral neuropathies. At present, it is thus thought that promotion of proper myelination, with few accompanying side effects as possible, is a potentially important therapeutic approach [3-5].

All stages of myelination by SCs are primarily accomplished by interactions of two different types of cells, namely, SCs and peripheral neurons [1,2]. While evidence illustrates that growth factors such as neuregulin-1 and insulin-like growth factor 1 (IGF1), as well as adhesion molecules, which are presented by peripheral neu- 
rons such as dorsal root ganglion (DRG) neurons, promote myelination [4-6]. It is unlikely that these growth factors and general signaling molecules that belong to downstream pathways provide suitable target molecules, since in most cases they are indispensable factors that support life. Here, we describe that in vivo knockdown of Dock7 in mice that have a specific short-hairpin RNA (shRNA) yields a specific phenotype with enhanced myelin thickness in SCs without observable reduced axon thickness in PNS sciatic nerves. We previously cloned the dock 7 gene and characterized Dock7 as a guanine-nucleotide exchange factor (GEF) that activates small GTPases of the Rho family, Rac1 and Cdc42 [7,8]. Rho GTPases are critical regulators that control cell morphological changes in many types of cells including SCs [9-13]. However, it remained unclear whether inhibition of Dock7 in vivo as well as in vitro affects myelination by SCs, or axon thickness in neurons, or both [8]. We for the first time report that Dock7 is an in vivo target molecule that specifically promotes SC myelination without reduced axon thickness, providing evidence that Dock7 may be an important therapeutic target for peripheral neuropathies that occur due to demyelination.

\section{MATERIALS AND METHODS}

\subsection{Antibodies}

The following antibodies were purchased: anti-Dock7 from Immuo-Biological Laboratories, Co., Ltd. (Fujioka, Gunma, Japan); anti-myelin basic protein (MBP) from Covance (Berkeley, CA, USA); and anti-actin from BD Biosciences Pharmingen (Franklin Lake, NJ, USA).

\subsection{Generation and Identification of shDock7 Transgenic Mice}

Genetically modified/unmodified mice were cared for in accordance with a protocol approved by the Japanese National Research Institute for Child Health and Development Animal Care Committee and were monitored by the Laboratory Animal Facility of the Japanese National Research Institute for Child Health and Development. First, the tandem units, namely, simian virus SV40 enhancer; artificial ferritin composite promoter, which combines human ferritin heavy chain FerH promoter with mouse elongation factor EF1 promoter, EGFP, and mouse EF1 polyadenylation signal, were amplified using the mouse in vivo expression vector $\mathrm{pVIVO} 2-\mathrm{GFP} / \mathrm{LacZ}$ (4982-9620 bases; InvivoGen, San Diego, CA, USA), as the template, and then digested with EcoRI and BamHI restriction enzymes. Second, the mouse shRNA transcription vector pSINsimU6 (1294-3944 bases; Takara Bio, Kyoto, Japan), which was inserted with ShDock7 oligonucleotide (sense oligonucleotide: 5'-GATCCGAC
GTTCGATGTCAATAGATAGTGCTCCTGGTTGTCT ATTGACATCGAACGTCTTTTTTAT-3'; antisense oligonucleotide: 5'-CGATAAAAAAGACGTTCGATGTC AATAGACAACCAGGAGCACTATCTATTGACATC GAACGTAG-3'), was digested with EcoRI. They were successively ligated into the EcoRI and BamHI sites of a pCMV5 backbone as the subcloning vector. The DNA fragment (approximately $6.2 \mathrm{~kb}$ ) containing EGFP and ShDock7 was digested from the vector backbone with NcoI, purified, and injected into fertilized BDF1 mouse oocytes $[8,14]$. Transgenic founders were mated to wild type C57BL/6JJmc and the transgene was stably maintained for at least 10 generations. Male mice were used for experiments when gender discrimination was possible. The transgenic mice and their nontransgenic littermates were fertile under standard breeding conditions. Identification of transgenic founder mice and transgenic mice was determined by PCR on genomic DNA prepared from tail biopsies (EGFP primer pair: 5'-CAATCATGA GCAAGGGAGAAGAACTCTTTACTGGTGTTGTC-3' and 5'-TTTACTTGTACAGCTCATCCATTCCCAGAG TAATTCCTGC-3'; and U6 primer pair: 5'-CGCACAG ACTTGTGGGAGAAGCTCGGCTACTC-3' and 5' - GC TTTGCATACTTCTGCCTGCTGGGGAGCCTG-3’). The primer pair for Oct $3 / 4$ as the positive control was 5 'CCGGGATCCAAGCTTTGTGAACTTGGCGGCTTCC AAGTCG-3' and 5'-CCGGGATCCCATTACTGGCCT GGTGCTTAGTTATCTTTG-3'. PCR was performed for 30 cycles, each consisting of denaturation at $94^{\circ} \mathrm{C}$ for 1 min, annealing at $65^{\circ} \mathrm{C}$ for $1 \mathrm{~min}$, and extension at $72^{\circ} \mathrm{C}$ for $1 \mathrm{~min}$. For Southern blotting, genomic DNAs were digested with EcoRI and BamHI and hybridized to a genomic probe for EGFP. The transgenic allele resulted in a hybridized band of $\sim 4.5 \mathrm{~kb}$.

\subsection{Cultures of Primary Cells and in Vitro Myelination}

Primary neurons were dissociated from dorsal root ganglia (DRGs) of C57BL/6JJms mouse embryos on embryonic day 13 (Sankyo Laboratories, Tokyo, Japan) and purified through culturing on collagen-coated dishes or glass coverslips in DMEM-GlutaMAX I (Life Technologies, Carlsbad, CA, USA) containing 10\% FBS, 100 $\mathrm{ng} / \mathrm{ml}$ NGF (AbD Serotec, Kidlington, UK), $8 \mu \mathrm{M}$ fluorodeoxyuridine, and $4 \mu \mathrm{M}$ uridine [15-17]. After 3 cycles of antimitotic reagent administration over $2-3$ weeks, purified DRG neurons were cultured in DMEMGlutaMAX I containing 10\% FBS and $100 \mathrm{ng} / \mathrm{ml}$ NGF. Primary Schwann cell precursors (SCPs) were also prepared from mouse DRGs on embryonic day 13 by the method of Ratner et al. [18] and cultured on polylysinecoated dishes in DMEM containing 10\% FBS, 1X B27, 10 ng/ml NRG1 (R \& D systems, Minneapolis, MN, USA), 
and $2 \mu \mathrm{M}$ forskolin. $\sim 200,000 \mathrm{SCs}$ were seeded onto purified DRG neuronal cultures $(\sim 70,000$ cells). SCneuronal cocultures were maintained in MEM containing $10 \%$ FBS and $100 \mathrm{ng} / \mathrm{ml}$ NGF [8]. Axonal processes and endogenous Schwann cells were allowed to grow and establish themselves for 5 days. For induction of myelination, $50 \mu \mathrm{g} / \mathrm{ml}$ ascorbic acid was added. Culture medium was changed every 2 to 3 days and cultures were maintained for an additional 10 days. Myelination was determined by immunostaining with an anti-MBP antibody.

\subsection{Immunofluorescence}

Cells on glass coverslips were fixed in PBS containing $4 \%$ paraformaldehyde and permeabilized with PBS containing $0.1 \%$ Tween-20. Permeabilized cells were blocked, and incubated first with a primary antibody and then with a fluorescence-labeled secondary antibody. The glass coverslips were mounted with Vectashield reagent (Vector Laboratories, Burlingame, CA, USA) onto slides for microscopic observation. The fluorescent images were captured using an Eclipse TE-300 microscope system (Nikon, Kawasaki, Japan) and analyzed with AxioVision software (Carl Zeiss, Oberkochen, Germany) or captured using a DMI4000 microscope system (Leica, Heerbrugg, Switzerland) and analyzed with AF6000 software (Leica).

\subsection{Electron Microscopy}

Sciatic nerves were fixed with $2 \%$ paraformaldehyde and $2 \%$ glutaraldehyde in $0.1 \%$ cacodylate buffer, contrasted with $2 \%$ osmium tetroxide, dehydrated with an ethanol gradient, and treated with propylene oxide. Finally, samples were infiltrated and embedded in pure epoxy. Ultrathin sections were stained with uranyl acetate and lead staining solution. Images were taken with a JEM-1200EX electron microscope system (JOEL, Tokyo, Japan).

\subsection{Immunoblotting}

Cells were lysed in lysis buffer A $(50 \mathrm{mM}$ HEPES$\mathrm{NaOH}, \mathrm{pH} 7.5,20 \mathrm{mM} \mathrm{MgCl} 2,150 \mathrm{mM} \mathrm{NaCl}, 1 \mathrm{mM}$ dithiothreitol, $1 \mathrm{mM}$ phenylmethane sulfonylfluoride, 1 $\mu \mathrm{g} / \mathrm{ml}$ leupeptin, $1 \mathrm{mM}$ EDTA, $1 \mathrm{mM} \mathrm{Na} \mathrm{VO}_{4}, 10 \mathrm{mM}$ $\mathrm{NaF}$, and $0.5 \%$ NP- 40 ) or lysis buffer A containing $1 \%$ CHAPS and $0.3 \%$ SDS. Unless otherwise indicated, all steps were performed at $4^{\circ} \mathrm{C}$. Proteins in the cell supernatants were denatured and then subjected to SDS-PAGE. The electrophoretically separated proteins were transferred to a PVDF membrane, blocked, and immunoblotted with a primary antibody and then with a peroxidaseconjugated secondary antibody. The bound antibodies were detected by chemiluminescence (Nacalai Tesque, Kyoto, Japan).

\subsection{Chromosome Preparation and Fluorescence in Situ Hybridization (FISH)}

Briefly, transgenic mouse splenocytes on glass slides were synchronized in the presence of thymidine for 16 hours and treated with 5-bromo-2'-deoxyuridine for an additional 5.5 hours. The cells were further treated with colcemid and harvested. Transgene-containing plasmid DNAs were labeled with Cy3-dUTP for use as probes. After hybridization, the glass slides were washed first in $50 \%$ formamide $/ 2 \times \mathrm{SSC}$ and then in $1 \times \mathrm{SSC}$, and counterstained with DAPI. FISH images were captured using a DMRA2 microscope system (Leica) and analyzed with CW4000 FISH software (Leica).

\subsection{Statistical Analysis}

Values shown represent the mean $\pm \mathrm{SD}$ from separate experiments. A one-way analysis of variance (ANOVA) was followed by a Fisher's protected least significant difference (PLSD) test as a post hoc comparison.

\section{RESULTS}

\subsection{Newly-Generated Dock7 shRNA Transgenic Mice Exhibit Enhanced Myelin Thickness without Affecting Neuronal Axon Diameter}

The aim of this study was to determine whether Dock7 has a specific effect on myelin sheaths formed by SCs but does not affect neuronal axon thickness in PNS sciatic nerves. To clarify this, we injected the transgene with Dock7 shRNA transcription sequence controlled under the U6 promoter and DNA sequence encoding EGFP as the genomic PCR marker into fertilized mouse oocytes (Figure 1(a)). Transgenic founders were mated to wild type mice and the transgene was stably maintained for at least 10 generations. The transgene was usually identified using genomic PCR (Figure 1(b)). Identification of the transgene by Southern blotting (Figure 1(c)) also showed that roughly 30 copies per genome were present. FISH analysis revealed that the transgene was inserted into the $\mathrm{B} 3$ to $\mathrm{C} 1$ regions of chromosome 12 (Figure 1(d)). These regions contain certain proteincoding genes and pseudogenes (see the Map Viewer website: http://www.ncbi.nlm.nih.gov/mapview), which seem unlikely to be positive regulators controlling changes in cell morphology. In primary SCs (Figure 2(a)) and sciatic nerves (Figure 2(b)) from Dock7 shRNA transgenic mice, Dock7 was effectively knocked down, as revealed by immunoblotting with an antibody specific for Dock7. Thus, we succeeded in generating novel Dock7 shRNA transgenic mice in which the transgene position differs from that of previously-characterized transgenic mice [8]. These transgenic mice, as well as their nontransgenic littermates, appeared healthy over 
(a)

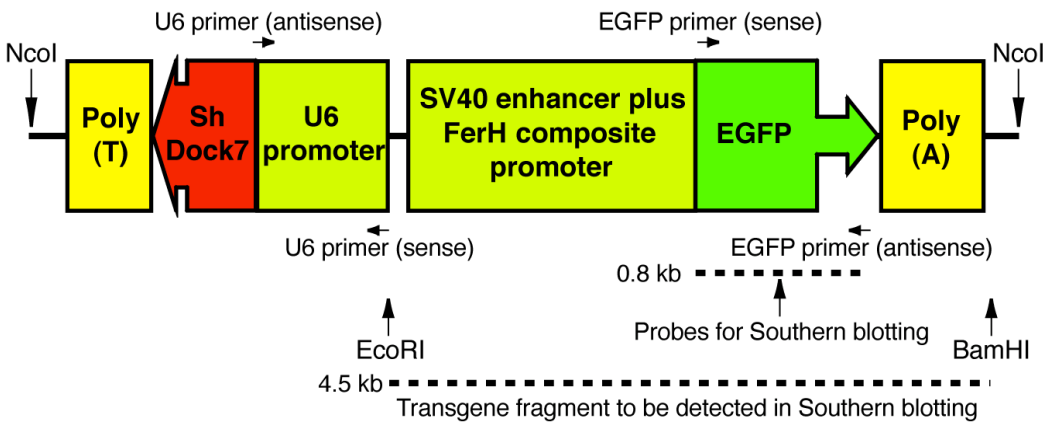

(b) Genomic PCR

(c) Southern blotting

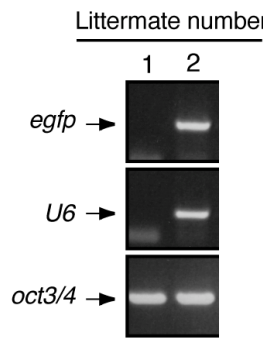

Positive control

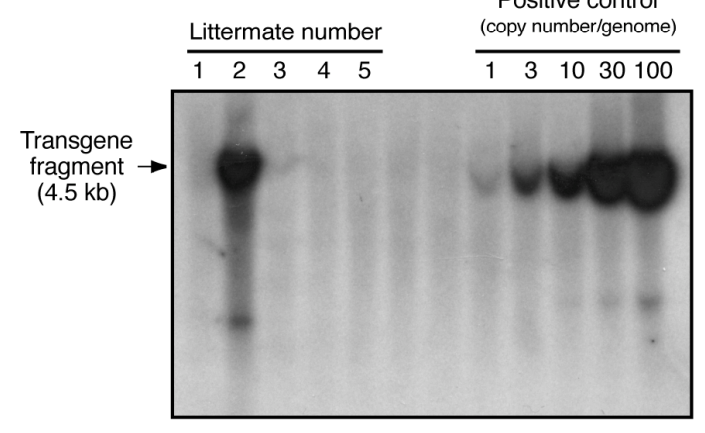

(d)

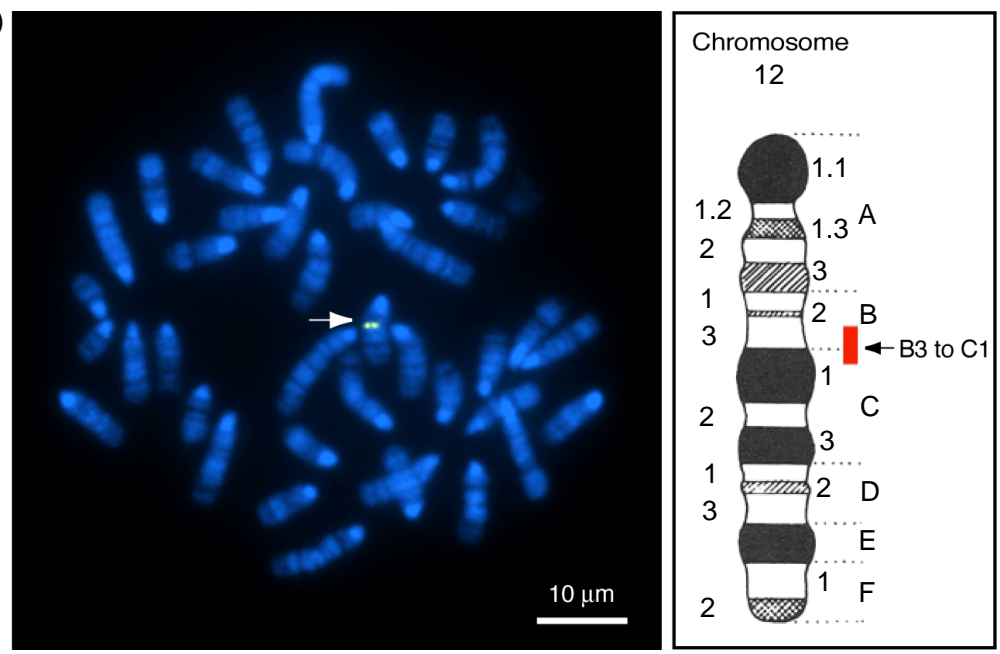

Figure 1. Transgenic mice have multiple copies of Dock7 shRNA transgenes in the B3 to $\mathrm{C} 1$ regions of chromosome 12. (a) The schematic structure of the transgene is shown; The positions of genomic PCR primers and the Southern blotting probe are also shown; (b) Genomic PCRs for the regions of the egfp gene and the U6 promoter were performed to determine transgene-positive mice. As the internal control, PCR amplification of the oct $3 / 4$ gene is also shown. In this time's experiment, a littermate corresponding to lane 1 was transgene-positive; (c) Southern blotting using egfp as the probe was performed. The numbers of transgene copies per genome was determined and compared with that of the positive controls. In this time's experiment, a littermate corresponding to lane 2 was transgene-positive and had roughly $\sim 30$ copies of transgenes per genome; (d) FISH analysis shows that the transgene is positioned in the B3 to C1 regions of mouse chromosome 12. The FISH-positive region is also shown in the schematic map of chromosome 12 .

one year and bred normally under standard breeding conditions.

To clarify whether knockdown of Dock7 has a specific effect on myelin formation in vivo, we analyzed sciatic nerve cross sections using electron microscopy. Dock7 shRNA transgenic mice exhibited enhanced myelin 
thickness compared with littermate controls (nontransgenic mice) and axon thickness in neurons was unaffected in both populations (Figures 3(a) and (b)). In fact, the average axon diameter in transgenic mice was $2.8 \mu \mathrm{m}$ $\pm 0.78 \mu \mathrm{m}(\mathrm{n}=52$ of the total number in two independent nerves) and that in the littermate controls was $2.7 \mu \mathrm{m}$ $\pm 0.73 \mu \mathrm{m}(\mathrm{n}=53$ of the total number in two independent nerves). The presence of myelinated axons with reduced diameters is the primary indicator of denatured neurons [3-5]. Rather, the average axon diameter in transgenic mice appeared to be slightly thicker than that in the controls. Additionally, 52\% of Dock 7 shRNA transgenic mice had myelinated axons with a sheath thickness greater than $1 \mu \mathrm{m}$ whereas only $32 \%$ of litter-

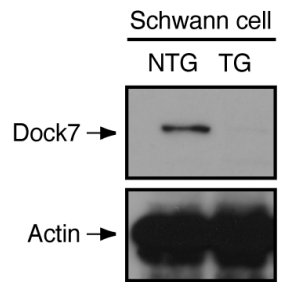

(a)

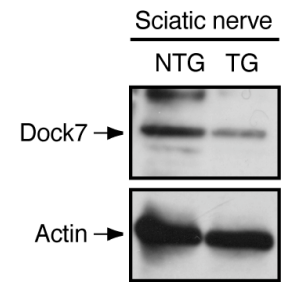

(b)
Figure 2. Expression of Dock7 protein is knocked down in Dock7 shRNA transgenic mice. (a) In the lysates from Dock7 shRNA transgenic (TG) mouse SCs and control littermate (nontransgenic, NTG) SCs, knockdown of Dock7 was confirmed by immunoblotting with an antibody specific for Dock7. As the internal control, expression of actin is also shown; (b) In the lysates from sciatic nerves, expression levels of Dock7 or actin was detected by immunoblotting with an antibody specific for Dock7 or actin, respectively.

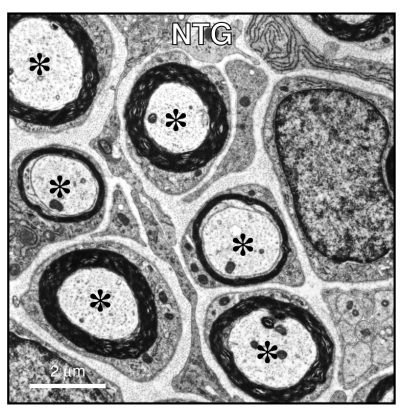

(a)

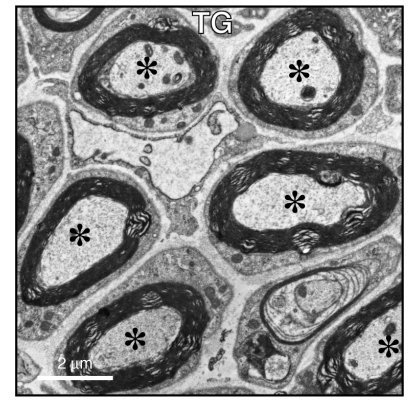

(b)
Figure 3. Dock 7 shRNA transgenic mice exhibit enhanced myelin thickness in SCs without affecting axon thickness in neurons in vivo. Representative sciatic nerve cross sections of Dock7 shRNA transgenic mice (a) and littermate controls (b) are shown. Dock7 shRNA transgenic mice exhibit enhanced myelin thickness compared with that of littermate controls. The average diameter of axons (indicated by asterisks) surrounded by multiply-layered myelin sheaths were comparable in transgenic mice and control mice. mate controls had myelin sheath thickness greater than 1 $\mu \mathrm{m}$ ( $\mathrm{n}=50$ for both). Taken together, these results suggest that knockdown of Dock7 in vivo has a specific effect on myelin thickness but not on reduction of axon thickness in sciatic nerves.

\subsection{Knockdown of Dock7 Promotes Myelination in Cocultures}

To confirm whether knockdown of Dock7 promotes myelination, we established SC-DRG neuronal cocultures. We isolated primary SCs from Dock7 shRNA transgenic mice and their littermate controls, and primary DRG neurons from wild type mice. SCs were cultured for proliferation in association with DRG axons, treated with ascorbic acid to initiate myelination, and allowed to wrap individual axons. Cultures were stained with an anti-MBP antibody to enable visualization of myelin segments. The representative images for myelin segments are depicted in Figures 4(a) and (b), showing that knockdown of Dock 7 promotes myelination compared with cocultures established from the SCs of littermate controls. In 200 $\mu \mathrm{m}$ microscopic square fields, Dock7 shRNA SC-neuronal cocultures showed $37 \pm 3.0$ of MBP-positive myelin segments whereas control SC-neuronal cocultures did only $7.3 \pm 1.9$ of segments $(\mathrm{n}=4, p<0.01)$. These results suggest that knockdown of Dock7 promotes myelination.

\section{DISCUSSION}

In this study, we succeeded in generating Dock 7 shRNA transgenic mice that exhibit enhanced myelin thickness in sciatic nerves without reduced axon diameters. We previously reported that Dock7 shRNA transgenic mice exhibit not only enhanced myelin thickness but also reduced axon diameter in neurons [8]. The reason for this discrepancy may be due to the difference in the transgene's position in the chromosome, unwelcome effect that is considered to be the major shortcoming of transgenic techniques applied in mice. The previous transgenic mice had the transgene in the B1 region of chromosome 12 [8], whereas the present transgenic mice have the transgene positioned in the $\mathrm{B} 3$ to $\mathrm{C} 1$ regions. The $\mathrm{B} 1$ region contains two protein-coding genes, namely, dock4 and zfp277 (the latter's function is unknown). Notably, Dock4 is a family member homologous to Dock7. Similar to Dock7, Dock4 acts as the GEF for small GTPases Rap1 and Rac1 [19,20] and plays a key role in neuronal development [20]. The transgene insertion may affect the expression of Dock4 protein to inhibit axon growth in neurons. Alternatively, the insertion may result in the expression of an incomplete form of Dock4 protein in neurons. Further investigation of the manner in which proteins involved in a transgene-in- 

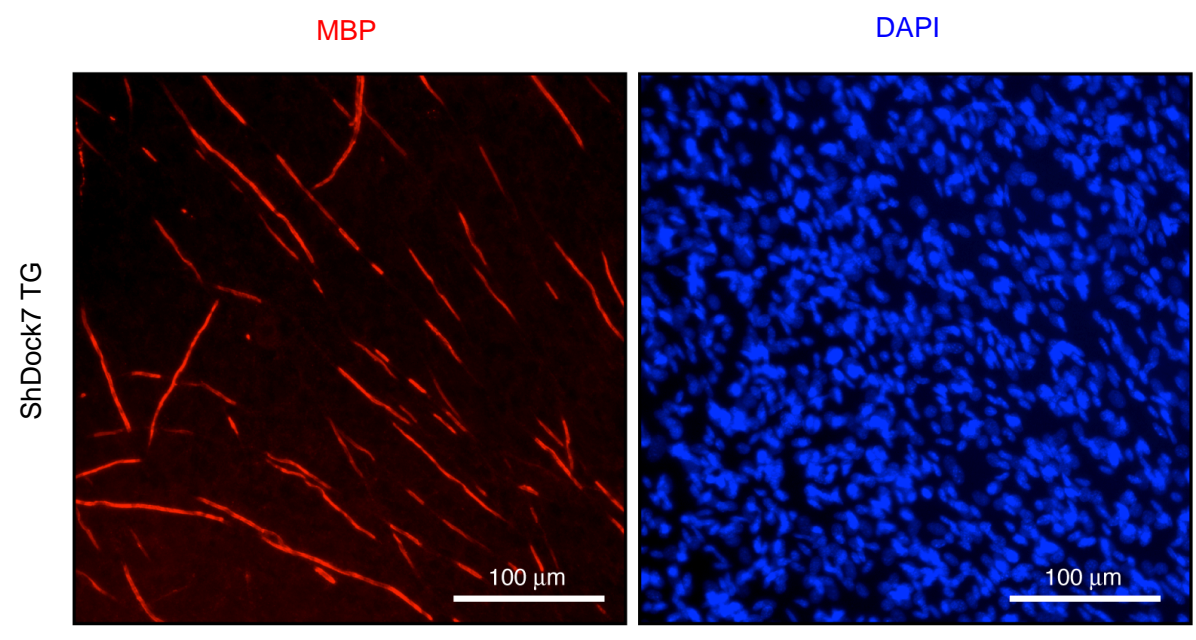

(a)
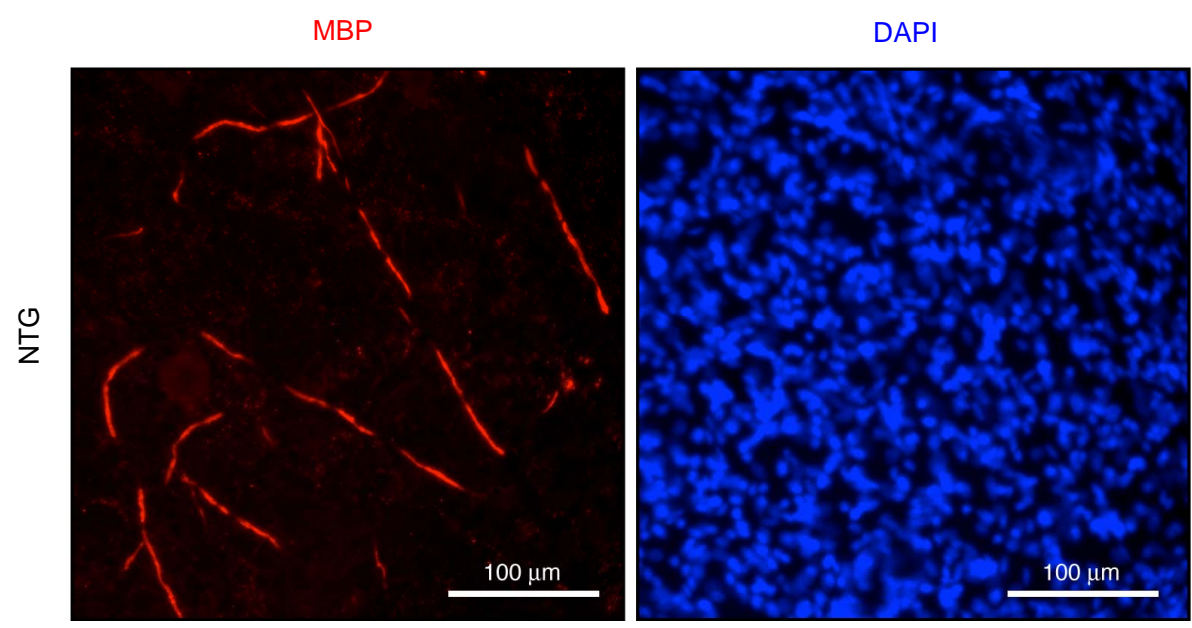

(b)

Figure 4. Coculture experiments show that Dock7 knockdown promotes myelination by SCs in vitro. (a) Primary SCs derived from Dock7 shRNA transgenic (TG) mice were cocultured with primary wild type DRG neurons and stained with an anti-MBP antibody to detect myelin segment, as well as DAPI to detect cocultured SCs; (b) SCs derived from the littermate controls (NTG) were cocultured with neurons and stained with an anti-MBP antibody. SC number was comparable in cultures established from TG and NTG. As shown, knockdown of Dock7 promotes myelination by SCs in vitro.

serted region are expressed may allow us to develop better mouse models for testing therapeutic target genes, as well as to generate mouse models using the knockout technique.

Most demyelinating diseases and related pathologies, especially in the PNS, show repeated demyelination and defective remyelination that exacerbates neuropathies. Therefore, finding therapeutic target molecules that promote proper myelination with minimal accompanying side effects may provide useful therapeutic methods for these diseases [3-5]. This study presents two important findings: 1) knockdown of Dock7 promotes myelination by SCs, and 2) this knockdown appears to have no reduced effect on axon thickness in neurons. Also, the transgenic mice in this study appeared to be healthy and bred normally. As far as we could ascertain, this is the first report of a target molecule that can specifically promote myelination without reduced axon thickness. Further studies on the role of Dock7 knockdown in pathological conditions may shed light on the development of novel therapeutic drug-target-specific medicines.

\section{ACKNOWLEDGEMENTS}

We thank Drs. W. Furmanski and K. Spicer for reading this manuscript. This work was supported by Grants-in-Aid for Scientific Research from the Japanese Ministry of Education, Culture, Sports, Science, and Technology (MEXT) and the Japanese Ministry of Health, Labor, and Welfare (MHLW) and partially supported by research grants from the 
Astellas Foundation, the Takeda Foundation, and the Uehara Foundation.

\section{REFERENCES}

[1] Bunge, R.P. (1993) Expanding roles for the Schwann cell: ensheathment, myelination, trophism and regeneration. Current Opinion in Neurobiology, 3, 805-809.

[2] Mirsky, R. and Jessen, K.R. (1996) Schwann cell development, differentiation and myelination. Current Opinion in Neurobiology, 6, 89-96. doi:10.1016/S0959-4388(96)80013-4

[3] Berger, P., Niemann, A. and Suter, U. (2006) Schwann cells and the pathogenesis of inherited motor and sensory neuropathies (Charcot-Marie-Tooth disease). Glia, 54, 243-257. doi:10.1002/glia.20386

[4] Nave, K.A. and Salzer, J.L. (2006) Axonal regulation of myelination by neuregulin 1. Current Opinion in Neurobiology, 16, 492-500. doi:10.1016/j.conb.2006.08.008

[5] Taveggia, C., Feltri, M.L. and Wrabetz, L. (2010) Signals to promote myelin formation and repair. Nature Reviews Neurology, 6, 276-287 doi:10.1038/nrneurol.2010.37

[6] Ogata, T., Iijima, S., Hoshikawa, S., Miura, T., Yamamoto, S., Oda, H., Nakamura, K. and Tanaka, S. (2004) Opposing extracellular signal-regulated kinase and Akt pathways control Schwann cell myelination. Journal of Neuroscience, 24, 6724-632. doi:10.1523/JNEUROSCI.5520-03.2004

[7] Yamauchi, J., Miyamoto, Y., Chan, J.R. and Tanoue, A. (2008) ErbB2 directly activates the exchange factor dock7 to promote Schwann cell migration. Journal of Cell Biology, 181, 351-365. doi:10.1083/jcb.200709033

[8] Yamauchi, J., Miyamoto, Y., Hamasaki, H., Sanbe, A., Kusakawa, S., Nakamura, A., Tsumura, H., Maeda, M., Nemoto, N., Kawahara, K., Torii, T. and Tanoue, A. (2011) The atypical guanine-nucleotide exchange factor, dock7, negatively regulates Schwann cell differentiation and myelination. Journal of Neuroscience, 31, 1257912592. doi:10.1523/JNEUROSCI.2738-11.2011

[9] Kaibuchi, K., Kuroda, S. and Amano, M. (1999) Regulation of the cytoskeleton and cell adhesion by the rho family GTPases in mammalian cells. Annual Review of Biochemistry, 68, 459-486. doi:10.1146/annurev.biochem.68.1.459

[10] Takai, Y., Sasaki, T. and Matozaki, T. (2001) Small GTPbinding proteins. Physiological Reviews, 81, 153-208.

[11] Schmidt, A. and Hall, A. (2002) Guanine nucleotide ex- change factors for rho GTPases: Turning on the switch. Genes \& Development, 16, 1587-1609. doi:10.1101/gad.1003302

[12] Rossman, K.L., Der, C.J. and Sondek, J. (2005) GEF means go: Turning on rho GTPases with guanine nucleotide-exchange factors. Nature Reviews Molecular Cell Biology, 6, 167-180. doi:10.1038/nrm1587

[13] Miyamoto, Y. and Yamauchi, J. (2010) The cellular signaling of dock family in neural function. Cell Signaling Technology, 22, 175-182. doi:10.1016/j.cellsig.2009.09.036

[14] Tanoue, A. Ito, S., Honda, K., Oshikawa, S., Kitagawa, Y., Koshimizu, T.A., Mori, T. and Tsujimoto, G. (2004) The vasopressin V1b receptor critically regulates hypothalamic-pituitary-adrenal axis activity under both stress and resting conditions. The Journal of Clinical Investigation, 113, 302-309. doi:10.1172/JCI200419656

[15] Yamauchi, J., Chan, J.R. and Shooter, E.M. (2004) Neurotrophins regulate Schwann cell migration by activating divergent signaling pathways dependent on rho GTPases. Proceedings of the National Academy of Sciences, 101, 8774-8779. doi:10.1073/pnas.0402795101

[16] Yamauchi, J., Chan, J.R., Miyamoto, Y., Tsujimoto, G. and Shooter, E.M. (2005) The neurotrophin-3 receptor TrkC directly phosphorylates and activates the nucleotide exchange factor Dbs to enhance Schwann cell migration. Proceedings of the National Academy of Sciences, 102, 5198-5203. doi:10.1073/pnas.0501160102

[17] Yamauchi, J., Miyamoto, Y., Tanoue, A., Shooter, E.M., and Chan, J.R. (2005b) Ras activation of a rac1 exchange factor, Tiam1, mediates neurotrophin-3-induced Schwann cell migration. Proceedings of the National Academy of Sciences, 102, 14889-14894. doi:10.1073/pnas.0507125102

[18] Ratner, N. Williams, J.P., Kordich, J.J. and Kim, H.A. (2006) Schwann cell preparation from single mouse embryos: Analyses of neurofibromin function in Schwann cells. Methods in Enzymology, 407, 22-33.

[19] Yajnik, V., Paulding, C., Sordella, R., McClatchey, A.I., Saito, M., Wahrer, D.C., Reynolds, P., Bell, D.W., Lake, R., van den Heuvel, S., Settleman, J. and Haber, D.A. (2003) Dock4, a GTPase activator, is disrupted during tumorigenesis. Cell, 112, 673-684. doi:10.1016/S0092-8674(03)00155-7

[20] Ueda, S., Fujimoto, S., Hiramoto, K., Negishi, M. and Katoh, H. (2008) Dock4 regulates dendritic development in hippocampal neurons. Journal of Neuroscience Research, 86, 3052-3061. doi:10.1002/jnr.21763 